FILE: 202005.tex, printed: 2020-7-31, 1.02

\title{
ON THE ORDER OF CONVEXITY FOR THE SHIFTED HYPERGEOMETRIC FUNCTIONS
}

\author{
LI-MEI WANG
}

\begin{abstract}
In the present paper, we study the order of convexity of $z_{2} F_{1}(a, b ; c ; z)$ with real parameters $a, b$ and $c$ where ${ }_{2} F_{1}(a, b ; c ; z)$ is the Gaussian hypergeometric function. First we obtain some conditions for $z_{2} F_{1}(a, b ; c ; z)$ with no any finite orders of convexity by considering its asymptotic behavior around $z=1$. Then the order of convexity of $z_{2} F_{1}(a, b ; c ; z)$ is demonstrated for some ranges of real parameters $a, b$ and $c$. In the last section, we give some examples as the applications of the main results.
\end{abstract}

\section{INTRODUCTION AND MAIN RESULTS}

The Gaussian hypergeometric function plays an important role in the special function theory and is related to many elementary functions. It is connected with conformal mappings, quasiconformal theory, differential equations, continued fractions and so on. For complex parameters $a, b, c(c \neq 0,-1,-2, \ldots)$, the hypergeometric function is defined by the power series

$$
{ }_{2} F_{1}(a, b ; c ; z)=\sum_{n=0}^{\infty} \frac{(a)_{n}(b)_{n}}{(c)_{n} n !} z^{n}
$$

for $z \in \mathbb{D}=\{z \in \mathbb{C}:|z|<1\}$, where $(a)_{n}$ is the Pochhammer symbol; namely, $(a)_{0}=1$ and $(a)_{n}=a(a+1) \cdots(a+n-1)=\Gamma(a+n) / \Gamma(a)$ for $n=1,2, \ldots$ If $c=a+b$, the hypergeometric function is termed as the zero-balanced one. Note that $z_{2} F_{1}(a, b ; c ; z)$ is usually called a shifted hypergeometric functions. For instance $z_{2} F_{1}(1,1 ; 2 ; z)=-\log (1-$ $z)$ is a shifted zero-balanced hypergeometric function. In the present paper, we only restrict to the real parameters $a, b$ and $c$. For the basic properties of hypergeometric functions we refer to (1), (8) and (17).

The behavior of the hypergeometric function ${ }_{2} F_{1}(a, b ; c ; z)$ near $z=1$ is completely different according to the sign of $a+b-c$, namely,

(i) For $a+b-c<0$

$$
{ }_{2} F_{1}(a, b ; c ; 1)=\frac{\Gamma(c) \Gamma(c-a-b)}{\Gamma(c-a) \Gamma(c-b)}<\infty
$$

(ii) For $a+b-c=0$

$$
{ }_{2} F_{1}(a, b ; a+b ; z) \sim-\frac{\Gamma(a) \Gamma(b)}{\Gamma(a+b)} \log (1-z), \text { as } z \rightarrow 1 .
$$

2010 Mathematics Subject Classification. Primary 30C45; Secondary 33C05.

Key words and phrases. Gaussian hypergeometric functions, order of convexity.

This research is supported by "the Fundamental Research Funds for the Central Universities" in UIBE (No. 18YB02) and National Natural Science Foundation of China (No. 11901086). 
(iii) For $a+b-c>0$

$$
{ }_{2} F_{1}(a, b ; c ; z) \sim \frac{\Gamma(c) \Gamma(a+b-c)}{\Gamma(a) \Gamma(b)}(1-z)^{c-a-b}, \text { as } z \rightarrow 1 .
$$

For more details see (3), (8), (9) and the references therein.

For a function $f$ analytic in $\mathbb{D}$ and normalized by $f(0)=f^{\prime}(0)-1=0$, the order of convexity of $f$ is defined by

$$
\kappa=\kappa(f):=1+\inf _{z \in \mathbb{D}} \operatorname{Re} \frac{z f^{\prime \prime}(z)}{f^{\prime}(z)} \in[-\infty, 1] .
$$

It is known that $f$ is convex, i.e. $\kappa(f) \geq 0$ if and only if $f$ is univalent in $\mathbb{D}$ and $f(\mathbb{D})$ is a convex domain. It is also true that if $\kappa(f) \geq-1 / 2$, then $f$ is univalent in $\mathbb{D}$ and $f(\mathbb{D})$ is convex in (at least) one direction, see (16) and (12, p.17, Thm.2.24; p.73). We make the convention that $\kappa(f)=-\infty$ only if $f^{\prime}$ has no zeros in $\mathbb{D}$ and $\operatorname{Re}\left[z f^{\prime \prime}(z) / f^{\prime}(z)\right]$ is not bounded from below in $\mathbb{D}$, whereas $\kappa(f)$ is regarded to be not defined if $f^{\prime}$ has zeros in $\mathbb{D}$.

The convexity of the shifted hypergeometric functions has been researched several times in the existing literature. For instance, Silverman constructed some sufficient conditions of convexity on the coefficients of the MacLaurin series in (14); Sugawa and the author got a condition for the convexity of a special shifted hypergeometric function with complex parameters by Jack's Lemma in (15); Ponnusamy and Vuorinen in (10) gave several necessary conditions for convexity by considering the bounds of the modulus of the $n$-th coefficients of the MacLaurin series; Küstner in (므) obtained the order of convexity of $z_{2} F_{1}(1, b ; c ; z)$ and $z_{2} F_{1}(a, b ; 2 ; z)$ for some special cases by transforming the convexity to the starlikeness of $z_{2} F_{1}(2, b ; c ; z)$ and $z_{2} F_{1}(a, b ; 1 ; z)$ respectively. We only state one of Küstner's results here, because it is closely related to our main results.

Theorem A ((마), Corollary 8).

(a) If $0<a \leq b \leq 1$ then

$$
\kappa\left(z_{2} F_{1}(a, b ; 2 ; z)\right)=1-\frac{{ }_{2} F_{1}{ }^{\prime}(a, b ; 1 ;-1)}{{ }_{2} F_{1}(a, b ; 1 ;-1)} .
$$

(b) If $0<-a \leq b \leq 1$ then

$$
\kappa\left(z_{2} F_{1}(a, b ; 2 ; z)\right)=1+\frac{{ }_{2} F_{1}{ }^{\prime}(a, b ; 1 ; 1)}{{ }_{2} F_{1}(a, b ; 1 ; 1)}=-\infty .
$$

(c) If $0<b<-a \leq 1$ then

$$
\kappa\left(z_{2} F_{1}(a, b ; 2 ; z)\right)=1+\frac{{ }_{2} F_{1}{ }^{\prime}(a, b ; 1 ; 1)}{{ }_{2} F_{1}(a, b ; 1 ; 1)}=1-\frac{a b}{a+b} .
$$

(d) If $0<a<1<b \leq 2-a$ then

$$
\kappa\left(z_{2} F_{1}(a, b ; 2 ; z)\right)=-\infty .
$$

(e) If $0<a \leq 1 \leq b \leq 2<a+b$ then

$$
\kappa\left(z_{2} F_{1}(a, b ; 2 ; z)\right)=1+\frac{(1-a)(1-b)}{a+b-2}+\frac{1-a-b}{2} .
$$


By observing the behavior of hypergeometric function around $z=1 \mathrm{in} \mathbb{D}$, we derive some conditions for the shifted hypergeometric function to have $-\infty$ as its order of convexity.

Theorem 1.1. For real parameters $a, b$ and $c$ none of which are negative integers satisfying $c-a \notin-\mathbb{N}$ and $c-b \notin-\mathbb{N}$, if one of the following conditions holds:

(1) $0<a b<1$ and $a+b \leq c<1+a+b-a b$;

(2) $a b<0$ and $a+b \leq c<1+a+b$;

then the order of convexity of the function $z_{2} F_{1}(a, b ; c ; z)$ is $-\infty$.

Note that Theorem 1.1 generalizes the case (d) in Theorem A and a result due to Küstner in (흐, Corollary 9, case (e)). Letting $c=a+b$ in Theorem 1.1, we obtain a result on the nonconvexity of the zero-balanced hypergeometric functions as follows.

Corollary 1.2. If $a$ and $b$ are real constants satisfying $a \neq 0,-1,-2, \cdots, b \neq 0,-1,-2, \cdots$ and $a b<1$, then the shifted zero-balanced hypergeometric function $z_{2} F_{1}(a, b ; a+b ; z)$ is not convex.

By applying the continued fraction representations of the ratio of two hypergeometric functions, we get the following results on the order of convexity of the shifted hypergeometric functions.

Theorem 1.3. Suppose $b$ and $c$ are real parameters with $0<b \leq c$.

(1) If $c \geq 2$, then

$$
\kappa\left(z_{2} F_{1}(1, b ; c ; z)\right)=\frac{4-b-c}{2}+\frac{c-2}{2} \frac{{ }_{2} F_{1}(1, b ; c ;-1)}{{ }_{2} F_{1}(2, b ; c ;-1)} .
$$

(2) If $1 \leq c<\min \{2,1+b\}$, then

$$
\kappa\left(z_{2} F_{1}(1, b ; c ; z)\right)=\frac{(c-b)(c+b-3)}{2(1+b-c)} .
$$

It is worth to point that the order of convexity of $z_{2} F_{1}(1, b ; c ; z)$ with real parameters $b$ and $c$ satisfying $0 \leq b \leq c$ and $1+b \leq c<2$ is already shown in Theorem 1.1.

Remark 1.4. Theorem 1.3 is also proved by Küstner in (6), Corollary 9, cases (a) and $(f))$ by the relationship between the starlikeness of $z_{2} F_{1}(2, b ; c ; z)$ and the convexity of $z_{2} F_{1}(1, b ; c ; z)$, although the order in the first case is given in different forms.

Theorem 1.5. For real parameters $a, b$ and $c$ satisfying $0<a<1, a \leq c$ and $0 \leq b \leq c$, the following hold for the order of convexity of the function $z_{2} F_{1}(a, b ; c ; z)$ :

(1) If $1-b>0$ and $c-2+(1-a)(1-b) \geq 0$, then

$$
\kappa\left(z_{2} F_{1}(a, b ; c ; z)\right)=\frac{5-c-a-b}{2}+M(-1) .
$$

(2) If $1-b<0$ and $c-2+(1-a)(1-b) \leq 0$, then

$$
\kappa\left(z_{2} F_{1}(a, b ; c ; z)\right)=\frac{5-c-a-b}{2}+M(1) .
$$


Here

$$
M(z)=\frac{c-2+(1-a)(1-b) z}{(1-z)\left(1-a+a \frac{{ }_{2} F_{1}(a+1, b ; c ; z)}{{ }_{2} F_{1}(a, b ; c ; z)}\right)} .
$$

Note that $M(1)$ in Theorem 1.5 is regarded as the (unrestricted) limit of $M(z)$ as $z \rightarrow 1$ in $\mathbb{D}$.

Remark 1.6. Note that if $b=1$ in Theorem 1.5, the order of convexity is already given in Theorem 1.3 since the hypergeometric function ${ }_{2} F_{1}(a, b ; c ; z)$ is symmetric in $a$ and $b$. If we let $c=2$ in Theorem [1.5, the first case is reduced to the cases (a) in Theorem A, even though the order is demonstrated in different forms.

A direct use of Theorem 1.5 and Lemma 2.2 yields the result on the lower orders of convexity for some special hypergeometric functions.

Corollary 1.7. For $0<a<1, a \leq c$ and $0<b \leq \min \{1, c\}$, then the order of convexity for the shifted hypergeometric function $z_{2} F_{1}(a, b ; c ; z)$ satisfies

$$
\kappa \geq\left\{\begin{array}{l}
\frac{(4-a b) c-a b(5-a-b)}{2(2 c-a b)}, c \geq 3-a-b+a b \\
\frac{2 c+\left(a^{2}-5 a+2\right) b}{2(b+c-a b)}, 1+a+b-a b \leq c<3-a-b+a b .
\end{array}\right.
$$

From Theorem 1.5 and Lemma 2.3, we can deduce the next consequence concerning the explicit order of convexity for the shifted hypergeometric functions with special parameters.

Corollary 1.8. For $0<a<1<b \leq c<\min \{a+b, 1+a+b-a b\}$, then the order of convexity for the shifted hypergeometric function $z_{2} F_{1}(a, b ; c ; z)$ is

$$
\frac{c^{2}-a^{2}-b^{2}+3(a+b-c)-2}{2(a+b-c)} .
$$

Remark 1.9. Put $c=2$ in Corollary 1.8 , then the result becomes the case (e) in Theorem $A$, since the condition $0<a<1<b \leq 2$ implies $1+a+b-a b>2$.

We can infer from Corollary 1.7 and Corollary 1.8 the convexity and non-convexity of the shifted hypergeometric function $z_{2} F_{1}(a, b ; c ; z)$, by analyzing the sign of the orders in these two corollaries.

Corollary 1.10. (1) The shifted hypergeometric function $z_{2} F_{1}(a, b ; c ; z)$ is convex in $\mathbb{D}$ if $0<a<1,0<b<1$ and $c \geq 1+a+b-a b$.

(2) For real parameters $a, b$ and $c$ with $0<a<1<b \leq c$, the shifted hypergeometric function $z_{2} F_{1}(a, b ; c ; z)$ is not convex in $\mathbb{D}$ if one of the following conditions holds:

(a) $a b<1$ and $c<a+b$;

(b) $a b>1$ and $c<\min \left\{1+a+b-a b, \frac{3+\sqrt{9+4\left(a^{2}+b^{3}-3 a-3 b+2\right)}}{2}\right\}$. 


\section{Some Lemmas}

This section is devoted to introducing several lemmas for later use.

Lemma 2.1 ((5) $)$, Thm. 1.5, (17), p.337-339 and Thm. 69.2). If $-1 \leq a \leq c$ and $0 \leq b \leq c \neq 0$, the ratio of two hypergeometric functions can be written in continued fraction and integral as

$$
\frac{{ }_{2} F_{1}(a+1, b ; c ; z)}{{ }_{2} F_{1}(a, b ; c ; z)}=\frac{1}{1-\frac{\left(1-g_{0}\right) g_{1} z}{1-\frac{\left(1-g_{1}\right) g_{2} z}{{ }_{1-}}}}=\int_{0}^{1} \frac{d \mu(t)}{1-t z}, \quad z \in \mathbb{C} \backslash[1,+\infty)
$$

where

$$
g_{n}= \begin{cases}0 & \text { for } n=0 \\ \frac{a+k}{c+2 k-1} & \text { for } n=2 k \geq 2, k \geq 1 \\ \frac{b+k-1}{c+2 k-2} & \text { for } n=2 k-1 \geq 1, k \geq 1\end{cases}
$$

and $\mu:[0,1] \rightarrow[0,1]$ is nondecreasing with $\mu(1)-\mu(0)=1$. Thus it is holomorphic in $\mathbb{C} \backslash[1,+\infty)$ and it maps the unit disk and the half plane $\{z \in \mathbb{C}: \operatorname{Re} z<1\}$ univalently onto domains that are convex in the direction of the imaginary axis.

Since the values of the hypergeometric functions at $z= \pm 1$ appear in Theorem 1.3 and Theorem 1.5, the next two lemmas deal with the estimations at $z=-1$ and the behaviors around $z=1$ respectively.

Lemma 2.2. If $-1 \leq a \leq c$ and $0 \leq b \leq c \neq 0$, then

$$
\frac{c}{b+c} \leq \frac{{ }_{2} F_{1}(a+1, b ; c ;-1)}{{ }_{2} F_{1}(a, b ; c ;-1)} \leq \frac{2 c-b}{2 c} .
$$

Proof. (1) Since $-1 \leq a \leq c$ and $0 \leq b \leq c \neq 0$, Lemma 2.1 implies that

$$
\frac{{ }_{2} F_{1}(a+1, b ; c ;-1)}{{ }_{2} F_{1}(a, b ; c ;-1)}=\frac{1}{1+\frac{\left(1-g_{0}\right) g_{1}}{1+\frac{\left(1-g_{1}\right) g_{2}}{\cdot}}}
$$

where $\left\{g_{n}\right\}_{n=0}^{\infty}$ is a nonnegative sequence shown in (2.1). Thus it is obvious that the above continued fraction is not less that $\frac{1}{1+\left(1-g_{0}\right) g_{1}}=\frac{c}{b+c}$. On the other hand, Theorem $11.1 \mathrm{in}$ (17, P.46) demonstrates that the values of the continued fraction

$$
\frac{g_{1}}{1-\frac{\left(1-g_{1}\right) g_{2} z}{1-\frac{\left(1-g_{2}\right) g_{3} z}{1-}}}
$$

lie in the circle

$$
\left|w-\frac{1}{2-g_{1}}\right| \leq \frac{1-g_{1}}{2-g_{1}}
$$

Therefore

$$
\frac{1}{1+\frac{\left(1-g_{0}\right) g_{1}}{1+\frac{\left(1-g_{1}\right) g_{2}}{1+}}} \leq \frac{1}{1+\frac{g_{1}}{2-g_{1}}}=\frac{2 c-b}{2 c} \text {. }
$$


We verify the assertions.

Lemma 2.3. Let $a, b$ and $c$ be real constants with $a, b, c \notin-\mathbb{N}, c-a \notin-\mathbb{N}$ and $c-b \notin-\mathbb{N}$.

(1) If $a+b<c<a+b+1$, then

$$
\frac{{ }_{2} F_{1}(a+1, b ; c ; z)}{{ }_{2} F_{1}(a, b ; c ; z)}=\frac{A}{(1-z)^{1-\alpha}}+O\left(|1-z|^{\varepsilon-1}\right)
$$

where

$$
A=\frac{\Gamma(a+b+1-c) \Gamma(c-a) \Gamma(c-b)}{\Gamma(a+1) \Gamma(b) \Gamma(c-a-b)},
$$

$\alpha=c-a-b \in(0,1)$ and $\varepsilon=\min \{2 \alpha, 1\}$.

(2) If $a+b=c$, then

$$
\frac{{ }_{2} F_{1}(a+1, b ; c ; z)}{{ }_{2} F_{1}(a, b ; c ; z)}=\frac{1}{-a(1-z) \log (1-z)}+O\left(\log \frac{1}{|1-z|}\right) .
$$

(3) If $c<a+b$, then

$$
\frac{{ }_{2} F_{1}(a+1, b ; c ; z)}{{ }_{2} F_{1}(a, b ; c ; z)}=\frac{a+b-c}{a(1-z)}+O\left(|1-z|^{a+b-c}\right) .
$$

Proof. (1) Since $a+b<c<a+b+1$, by applying the formula

$$
\begin{aligned}
& { }_{2} F_{1}(a, b ; c ; z)=\frac{\Gamma(c) \Gamma(c-a-b)}{\Gamma(c-a) \Gamma(c-b)}{ }_{2} F_{1}(a, b ; a+b-c+1 ; 1-z) \\
& \quad+(1-z)^{c-a-b} \frac{\Gamma(c) \Gamma(a+b-c)}{\Gamma(a) \Gamma(b)}{ }_{2} F_{1}(c-a, c-b ; c-a-b+1 ; 1-z)
\end{aligned}
$$

to the functions ${ }_{2} F_{1}(a+1, b ; c ; z)$ and ${ }_{2} F_{1}(a, b ; c ; z)$, we have for $z \rightarrow 1$ in $\mathbb{D}$,

$$
\begin{aligned}
& \frac{{ }_{2} F_{1}(a+1, b ; c ; z)}{{ }_{2} F_{1}(a, b ; c ; z)} \\
= & (1-z)^{c-a-b-1} \frac{\Gamma(c) \Gamma(a+b+1-c)}{\Gamma(a+1) \Gamma(b)} \frac{\Gamma(c-a) \Gamma(c-b)}{\Gamma(c) \Gamma(c-a-b)}+O\left(|1-z|^{\varepsilon-1}\right) \\
= & (1-z)^{c-a-b-1} \frac{\Gamma(a+b+1-c) \Gamma(c-a) \Gamma(c-b)}{\Gamma(a+1) \Gamma(b) \Gamma(c-a-b)}+O\left(|1-z|^{\varepsilon-1}\right) \\
:= & A(1-z)^{\alpha-1}+O\left(|1-z|^{\varepsilon-1}\right)
\end{aligned}
$$

where

$$
A=\frac{\Gamma(a+b+1-c) \Gamma(c-a) \Gamma(c-b)}{\Gamma(a+1) \Gamma(b) \Gamma(c-a-b)}>0,
$$

$\varepsilon=\min \{2(c-a-b), 1\}$ and $\alpha=c-a-b \in(0,1)$ since $a+b<c<a+b+1$.

(2) If $c=a+b$, by virtue of (2.5) and the following formula due to Ramanujan

$$
{ }_{2} F_{1}(a, b ; a+b ; z)=\frac{\Gamma(c)}{\Gamma(a) \Gamma(b)}(R(a, b)-\log (1-z))+O\left(|1-z| \log \frac{1}{|1-z|}\right)
$$

as $z \rightarrow 1$ in $\mathbb{D}$, where

$$
R(a, b)=2 \psi(1)-\psi(a)-\psi(b)
$$


and $\psi(x)=\Gamma^{\prime}(x) / \Gamma(x)$ denotes the digamma function, we have around $z=1$,

$$
\begin{aligned}
& \frac{{ }_{2} F_{1}(a+1, b ; c ; z)}{{ }_{2} F_{1}(a, b ; c ; z)} \\
= & (1-z)^{-1} \frac{\Gamma(a+b) \Gamma(1)}{\Gamma(a+1) \Gamma(b)} \times \frac{\Gamma(a) \Gamma(b)}{-\log (1-z) \Gamma(a+b)}+O\left(\log \frac{1}{|1-z|}\right) \\
= & \frac{1}{-a(1-z) \log (1-z)}+O\left(\log \frac{1}{|1-z|}\right) .
\end{aligned}
$$

(3) Since $c<a+b$, we deduce from the transformation (2.5) that around $z=1$,

$$
\begin{aligned}
& \frac{{ }_{2} F_{1}(a+1, b ; c ; z)}{{ }_{2} F_{1}(a, b ; c ; z)} \\
= & (1-z)^{-1} \frac{\Gamma(a+b+1-c)}{\Gamma(a+1) \Gamma(b)} \times \frac{\Gamma(a) \Gamma(b)}{\Gamma(a+b-c)}+O\left(|1-z|^{a+b-c}\right) \\
= & \frac{a+b-c}{a(1-z)}+O\left(|1-z|^{a+b-c}\right) .
\end{aligned}
$$

Lemma $2.4((\underline{13}))$. Let $F(z)$ be analytic in the slit domain $\mathbb{C} \backslash[1,+\infty)$. Then

$$
F(z)=\int_{0}^{1} \frac{d \mu(t)}{1-t z}
$$

for some probability measure $\mu$ on $[0,1]$, if and only if the following conditions are fulfilled:

(1) $F(0)=1$;

(2) $F(x) \in \mathbb{R}$ for $x \in(-\infty, 1)$;

(3) $\operatorname{Im} F(z) \geq 0$ for $\operatorname{Im} z>0$;

(4) $\lim _{n \rightarrow \infty} F\left(z_{n}\right) / z_{n}=0$ for some sequence $z_{n} \in \mathbb{C}$ with $\operatorname{Im} z_{n} \rightarrow+\infty$, and $\operatorname{Im} z_{n} \geq$ $\delta \operatorname{Re} z_{n}$ for some positive constant $\delta$;

(5) $\limsup _{x \rightarrow+\infty} F(-x) \geq 0$.

The measure $\mu$ and the functions $F$ are in one-to-one correspondence.

It is noteworthy that Liu and Pego in (7) proved that the condition (4) in Lemma 2.4 is superfluous.

The next lemma is a direct consequence of Lemma 2.4 .

Lemma 2.5. If

$$
f(z)=\int_{0}^{1} \frac{d \mu(t)}{1-t z}
$$


for some probability measure $\mu$ on $[0,1]$, then for any $0<a \leq 1$, there exists some probability measure $\nu$ on $[0,1]$ such that

$$
\frac{1}{(1-z)(1-a+a f)}=\int_{0}^{1} \frac{d \nu(t)}{1-t z} .
$$

\section{Proofs of the MAIN RESUlts}

Before proceeding to prove the main results, we first prepare some materials which will be used several times in the proofs.

Let $F(z)={ }_{2} F_{1}(a, b ; c ; z), G(z)={ }_{2} F_{1}(a+1, b ; c ; z)$ and $H(z)={ }_{2} F_{1}(a+1, b+1 ; c+1 ; z)$ for simplicity. Contiguous relations of hypergeometric functions imply that

$$
G(z)-F(z)=\frac{b}{c} z H(z)
$$

In order to obtain the order of convexity of $z F(z)$, we need to estimate the real part of $1+z(z F)^{\prime \prime}(z) /(z F)^{\prime}(z)$ in $\mathbb{D}$. By combining the derivative formula

$$
z F^{\prime}(z)=\frac{a b}{c} z H=-a F(z)+a G(z)
$$

and the hypergeometric differential equation

$$
z(1-z) F^{\prime \prime}(z)+[c-(a+b+1) z] F^{\prime}(z)-a b F(z)=0,
$$

a routine computation shows that

$$
\begin{aligned}
& 1+\frac{z(z F)^{\prime \prime}}{(z F)^{\prime}}=1+\frac{2 z F^{\prime}+z^{2} F^{\prime \prime}}{F+z F^{\prime}} \\
= & 1+\frac{z[2-c+(a+b-1) z] F^{\prime}+a b z F}{(1-z)\left(F+z F^{\prime}\right)} \\
= & \frac{a[3-c+(a+b-2) z](G-F)+[1+(a b-1) z] F}{(1-z)[F+a(G-F)]} \\
= & \frac{3-c+(a+b-2) z}{1-z}+\frac{c-2+(1-a)(1-b) z}{(1-z)(1-a+a G / F)} .
\end{aligned}
$$

Denote

$$
W(z)=\frac{3-c+(a+b-2) z}{1-z}+M(z)
$$

with

$$
M(z)=\frac{c-2+(1-a)(1-b) z}{(1-z)(1-a+a G / F)}
$$

If $0 \leq a \leq \min \{c, 1\}$ and $0 \leq b \leq c$, a combination of Lemma 2.1 and Lemma 2.5 implies that there exists a probability measure $\nu$ on $[0,1]$ such that

$$
\frac{1}{(1-z)(1-a+a G / F)}=\int_{0}^{1} \frac{d \nu(t)}{1-t z} .
$$

Now we are ready to the proofs of the main results. 
Proof of Theorem 1.1. Since the first term in (3.2) has bounded real part in $\mathbb{D}$, it is sufficient to only consider the behavior of the second term $M(z)$. Rewrite

$$
M(z)=\frac{c-2+(1-a)(1-b) z}{(1-z)(1-a+a G / F)}=\frac{p}{(1-z)(1-a+a G / F)}-\frac{(1-a)(1-b)}{1-a+a G / F},
$$

with $p=c-1-a-b+a b$.

Both of the assumptions guarantee that $p<0$ and $a+b \leq c<a+b+1$. Thus the equations (2.2) and (2.3) in Lemma 2.3 imply that for $z \rightarrow 1$ in $\mathbb{D}$, the second term on the right hand side of $M(z)$ is bounded. To verify $\operatorname{Re} M(z)$ tends to $-\infty$ for $z \rightarrow 1$ in $\mathbb{D}$, we need only to analyze the asymptotic behavior of $(1-z)(1-a+a G / F)$ in the first term. Let $z_{\theta} \in \mathbb{D}$ with $z_{\theta}=1-r e^{i \theta}$, thus $-\pi / 2<\theta<\pi / 2$ and $0 \leq r<2 \cos \theta$. Since the asymptotic behaviors of the ratio of two hypergeometric functions $G / F$ around $z=1$ according to the sign of $c-a-b$, we divide the proof into two parts.

Case I: Let $a+b<c<1+a+b$. The equation (2.2) yields

$$
\begin{aligned}
& \tan \left[\arg \left(1-z_{\theta}\right)\left(1-a+a(G / F)\left(z_{\theta}\right)\right)\right] \\
= & \frac{\operatorname{Im}\left[(1-a) r e^{i \theta}+O\left(r^{\varepsilon}\right)+a A\left(r e^{i \theta}\right)^{\alpha}\right]}{\operatorname{Re}\left[(1-a) r e^{i \theta}+O\left(r^{\varepsilon}\right)+a A\left(r e^{i \theta}\right)^{\alpha}\right]} \\
= & \frac{(1-a) r \sin \theta+\operatorname{Im} O\left(r^{\varepsilon}\right)+a A r^{\alpha} \sin [\alpha \theta]}{(1-a) r \cos \theta+\operatorname{Re} O\left(r^{\varepsilon}\right)+a A r^{\alpha} \cos [\alpha \theta]} \\
= & \frac{(1-a) r^{1-\alpha} \sin \theta+\operatorname{Im} O\left(r^{\varepsilon-\alpha}\right)+a A \sin [\alpha \theta]}{(1-a) r^{1-\alpha} \cos \theta+\operatorname{Re} O\left(r^{\varepsilon-\alpha}\right)+a A \cos [\alpha \theta]} .
\end{aligned}
$$

Since $\alpha \in(0,1)$ and $\varepsilon>\alpha$, if we let $\theta \rightarrow \pm \pi / 2$ and $r \rightarrow 0$ correspondingly, then

$$
\arg \left(1-z_{\theta}\right)\left(1-a+a(G / F)\left(z_{\theta}\right)\right) \rightarrow \pm \frac{\alpha \pi}{2}
$$

and

$$
\mid\left(1-z_{\theta}\right)\left(1-a+a(G / F)\left(z_{\theta}\right) \mid \rightarrow 0 .\right.
$$

Finally we proved

$$
\operatorname{Re} \frac{1}{\left(1-z_{\theta}\right)\left(1-a+a(G / F)\left(z_{\theta}\right)\right)} \rightarrow+\infty
$$

as $\theta \rightarrow \pm \frac{\pi}{2}$.

Case II: If $a+b=c$, the equation (2.3) implies that

$$
\begin{aligned}
& \tan \arg \left[\left(1-z_{\theta}\right)\left(1-a+a(G / F)\left(z_{\theta}\right)\right)\right] \\
= & \tan \arg \left[\left(1-z_{\theta}\right)(1-a)-\frac{1}{\log \left(1-z_{\theta}\right)}+O\left(\left|1-z_{\theta}\right| \log \frac{1}{\left|1-z_{\theta}\right|}\right)\right] \\
= & \frac{(1-a) r \sin \theta+\frac{\theta}{\log ^{2} r+\theta^{2}}+\operatorname{Im} O(-r \log r)}{(1-a) r \cos \theta-\frac{\log r}{\log ^{2} r+\theta^{2}}+\operatorname{Re} O(-r \log r)} .
\end{aligned}
$$

After multiplying the term $\log ^{2} r$ in the denominator and numerator of the above equation, if we let $\theta \rightarrow \pm \pi / 2$ and $r \rightarrow 0$ correspondingly, then

$$
\arg \left(1-z_{\theta}\right)\left(1-a+a(G / F)\left(z_{\theta}\right)\right) \rightarrow 0
$$


and

$$
\mid\left(1-z_{\theta}\right)\left(1-a+a(G / F)\left(z_{\theta}\right) \mid \rightarrow 0,\right.
$$

from which we also deduce (3.5) in this case.

Therefore in both of these two cases, we have

$$
\operatorname{Re} \frac{p}{\left(1-z_{\theta}\right)\left(1-a+a(G / F)\left(z_{\theta}\right)\right)} \rightarrow-\infty
$$

as $\theta \rightarrow \pm \frac{\pi}{2}$, since $p<0$. The proof is complete.

Proof of Theorem 1.3. Since $a=1$, by making use of the representation (3.4), we can reform $W(z)$ as

$$
W(z)=\frac{3-c+(b-1) z}{1-z}+\frac{c-2}{(1-z) G / F}=\frac{3-c+(b-1) z}{1-z}+(c-2) \int_{0}^{1} \frac{d \nu(t)}{1-t z}
$$

where $\nu$ is a probability measure on $[0,1]$.

Thus if $c-2 \geq 0$, we have for $z \in \mathbb{D}$,

$$
\operatorname{Re} W(z) \geq W(-1)=\frac{4-b-c}{2}+\frac{c-2}{2} \frac{{ }_{2} F_{1}(1, b ; c ;-1)}{{ }_{2} F_{1}(2, b ; c ;-1)} .
$$

If $c-2<0$, it is obvious that

$$
\operatorname{Re} W(z) \geq \frac{4-b-c}{2}+(c-2) \lim _{\substack{z \rightarrow 1 \\ z \in \mathbb{D}}} \frac{{ }_{2} F_{1}(1, b ; c ; z)}{(1-z)_{2} F_{1}(2, b ; c ; z)} .
$$

On the other hand, the real part of the Möbius transform $(3-c+(b-1) z) /(1-z)$ can approach $(4-b-c) / 2$ as $z \rightarrow 1$ in $\mathbb{D}$. For example we can choose a sequence $z_{n}=1 / n+(1-1 / n) e^{i \pi / n} \in \mathbb{D}$, for $n \geq 2$. Since

$$
\operatorname{Re} \frac{3-c+(b-1) z_{n}}{1-z_{n}} \rightarrow \frac{4-b-c}{2}
$$

for $n \rightarrow \infty$, we get

$$
\min _{z \in \mathbb{D}} \operatorname{Re} W(z)=\frac{4-b-c}{2}+(c-2) \lim _{\substack{z \rightarrow 1 \\ z \in \mathbb{D}}} \frac{{ }_{2} F_{1}(1, b ; c ; z)}{(1-z){ }_{2} F_{1}(2, b ; c ; z)} .
$$

Furthermore if $c<\min \{2,1+b\}$, the equation (2.4) shows that

$$
\lim _{\substack{z \rightarrow 1 \\ z \in \mathbb{D}}} \frac{{ }_{2} F_{1}(1, b ; c ; z)}{(1-z){ }_{2} F_{1}(2, b ; c ; z)}=\lim _{\substack{z \rightarrow 1 \\ z \in \mathbb{D}}} \frac{1}{1+b-c+O\left(|1-z|^{2+b-c}\right)}=\frac{1}{1+b-c} .
$$

We finish the proof. 
Proof of Theorem 1.5. The representation (3.4) implies that there exists a probability measure $\nu$ on $[0,1]$ such that

$$
\begin{aligned}
M(z) & =\int_{0}^{1} \frac{c-2+(1-a)(1-b) z}{1-t z} d \nu(t) \\
& =c-2+\int_{0}^{1}[(1-a)(1-b)+(c-2) t] \frac{z}{1-t z} d \nu(t) .
\end{aligned}
$$

For fixed $t \in[0,1]$, the second factor of the integrant in the integral form of $M(z)$ is a Möbius transform which maps the unit disc $\mathbb{D}$ onto a disc or a half plane which is symmetric with respect to the real axis. Thus we arrive at the following claims.

(1) If $(1-a)(1-b)+(c-2) t \geq 0$ for all $t \in[0,1]$, i.e. $1-b \geq 0$ and $c-2+(1-a)(1-b) \geq 0$, then

$$
\min _{z \in \mathbb{D}} \operatorname{Re} M(z)=M(-1)
$$

thus

$$
\min _{z \in \mathbb{D}} \operatorname{Re} W(z)=\frac{5-a-b-c}{2}+M(-1) .
$$

(2) If $(1-a)(1-b)+(c-2) t \leq 0$ for all $t \in[0,1]$, i.e. $1-b \leq 0$ and $c-2+(1-a)(1-b) \leq 0$, then

$$
\min _{z \in \mathbb{D}} \operatorname{Re} M(z)=M(1)
$$

We further obtain that

$$
\min _{z \in \mathbb{D}} \operatorname{Re} W(z)=\frac{5-a-b-c}{2}+M(1),
$$

by the same sequence technique used in the proof of Theorem 1.3 since the first term of $W(z)$ also a Möbius transform.

Therefore we have all the assertions in this Theorem. The proof is completed.

\section{Some Examples and Remarks}

In this section, we find some applications of the main results, as well as some comparisons with the previous known results.

By specifying $(b, c)=(1,3),(b, c)=(3 / 2,3),(b, c)=(1,3 / 2)$ in Theorem 1.3 successively, we find the explicit order of convexity for some shifted hypergeometric functions.

Example 4.1. (1) The order of convexity of the function

$$
2+2 \frac{1-z}{z} \log (1-z)=z_{2} F_{1}(1,1 ; 3 ; z)
$$

is $\frac{\log 2}{2(2 \log 2-1)} \approx 0.8971$.

(2) The order of convexity of the function

$$
\frac{4 z}{(1+\sqrt{1-z})^{2}}=z_{2} F_{1}(1,3 / 2 ; 3 ; z)
$$

is $\frac{1+\sqrt{2}}{4} \approx 0.6035$.

(3) The order of convexity of the function $z_{2} F_{1}(1,1 ; 3 / 2 ; z)$ is $-1 / 4$. 
Remark 4.2. Put $c=1+b$ in Theorem 1.3, we arrive at the consequence that for $b \geq 1$, the function

$$
z_{2} F_{1}(1, b ; 1+b ; z)=\sum_{n=1}^{\infty} \frac{b}{b-1+n} z^{n}
$$

is convex of order $1 /(1+b)$. Note that the convexity of this function is contained in Ruscheweyh (11) and Sugawa and the author (15). In (15), the complex parameter $b$ is considered. Although here we only deal with the real one, the explicit order of convexity is given.

In view of Theorem 1.3 and Lemma 2.2 and Lemma 2.3, we obtain a result on the convexity of the shifted hypergeometric function.

Corollary 4.3. Assume $b$ and $c$ are real constants satisfying $0<b \leq c$, the function $z_{2} F_{1}(1, b ; c ; z)$ is convex if one of the following conditions holds:

(1) $0 \leq b \leq 1$ and $c \geq 2$;

(2) $1<b<2 \leq c<\frac{4 b-b^{2}}{2-2 b}$;

(3) $1 \leq c<\min \{2,1+b\}$ and $c \geq 3-b$.

Proof. In view of the different cases in Theorem 1.3, we divide the proof into two cases accordingly.

Case I: Let $c \geq 2$. Theorem 1.3 together with Lemma 2.2 shows that the order of convexity of the function $z_{2} F_{1}(1, b ; c ; z)$ satisfies

$$
\kappa \geq \frac{4-b-c}{2}+\frac{c-2}{2 c /(b+c)}=\frac{2 c+2 b(2-c)-b^{2}}{2(b+c)}:=\frac{L(b, c)}{2(b+c)} .
$$

Since $L_{c}^{\prime}(b, c)=2(1-b)$, the function $L(b, c)$ is nondecreasing and increasing in $c$ when $0 \leq b \leq 1$ and $b>1$ respectively.

For $0 \leq b \leq 1$ and $c \geq 2$, we deduce that $L(b, c)>0$ from the fact that $L(b, 2)>0$.

If $b>1$ and $2 \leq c \leq\left(4 b-b^{2}\right) /(2 b-2):=c_{0}$, then $L(b, c) \geq 0$ since $L\left(b, c_{0}\right)=0$.

Case II: Assume $1 \leq c<\min \{2,1+b\}$, then we infer from Theorem 1.3 that the order of convexity is

$$
\kappa=\frac{(c-b)(b+c-3)}{2(1+b-c)}
$$

which is obvious nonnegative if $c \geq 3-b$.

We verify all the cases of this theorem.

Recall that Hästö et al. in (4) proved that for nonzero real numbers $b$ and $c$, if $c \geq$ $\max \{3-b, 3 b\}$, then $z_{2} F_{1}(1, b ; c ; z)$ is a convex function. Therefore the above corollary partially generalizes their result.

Example 4.4. Let $(a, b, c)=(1 / 2,1 / 2,3)$ and $(a, b, c)=(1 / 2,1 / 2,2)$ in Corollary 1.7 and $(a, b, c)=(3 / 4,3 / 2,2)$ in Corollary 1.8 successively, we obtain that

(1) The order of convexity of the functions $z_{2} F_{1}(1 / 2,1 / 2 ; 3 ; z)$ and $z_{2} F_{1}(1 / 2,1 / 2 ; 2 ; z)$ are at least $41 / 46$ and $31 / 36$ respectively.

(2) The order of convexity of the function $z_{2} F_{1}(3 / 4,3 / 2 ; 2 ; z)$ is $-1 / 8$. 
Acknowledgements. The author would like to thank Professor Toshiyuki Sugawa for discussions and enlightening suggestions.

\section{REFERENCES}

1. M. Abramowitz and I. Stegun, Handbook of Mathematical Functions, Dover, New York, 1965.

2. A. Baken, S. Ruscheweyh and L. Salinas, Universal convexity and univeral starlikeness of Polylogarithms, Proc. Amer. Math. Soc. 143 (2015), 717-729.

3. R. J. Evans, Ramanujan's second notebook: asymptotic expansions for hypergeometric series and related functions, in G.E. Andrews, R.A. Askey, B.C. Berndt, R.G. Ramanathan, R.A. Rankin (Eds.), Ramanujan Revisited: Proc. Centenary Conf. Univ. of Illinois at Urbana-Champaign, Academic Press, Boston, 1988, 537-560.

4. P. Hästö, S. Ponnusamy and M. Vuorinen, Starlikeness of the Gaussian hypergeometric functions, Complex Var. Elliptic Equ. 55 (2010), 173184.

5. R. Küstner, Mapping properties of hypergeometric functions and convolutions of starlike or convex functions of order $\alpha$, Comput. Methods Funct. Theory, 2 (2002), 597610.

6. - On the order of starlikeness of the shifted Gauss hypergeometric function, J. Math. Anal. Appl. 334 (2007), 1363-1385.

7. Jian-Guo Liu and R. L. Pego, On generating functions of Hausdorff moment sequences, Trans. Amer. Math. Soc. 368 (2016), 8499-8518.

8. F. W. Olver, D. W. Lozier, R. F. Boisvert, and C. W. Clark, NIST Handbook of Mathematical Functions, Cambridge University Press, 2010.

9. S. Ponnusamy, Close-to-convexity properties of Gaussian hypergeometric functions, J. Comput. Appl. Math. 88 (1997), 327337.

10. S. Ponnusamy and M. Vuorinen, Univalence and convexity properties for Gaussian hypergeometric functions, Rocky Mountain J. Math. 31 (2001), 327-353.

11. St. Ruscheweyh, New criteria for univalent functions, Proc. Amer. Math. Soc. 49 (1975), 109-115.

12. __ Convolution in Geometric Function Theory, Sém. Math. Sup. 83, University of Montréal, Montréal, Québec, Canada 1982.

13. S. Ruscheweyh, L. Salinas and T. Sugawa, Completely monotone sequences and universally prestarlike functions, Isr. J. Math. 171(2009), 285-304.

14. H. Silverman, Starlike and convexity properties for hypergeometric functions, J. Math. Anal. Appl. 172 (1993), 574581.

15. T. Sugawa and L.-M.Wang, Spirallikeness of shifted hypergeometric functions, Ann. Acad. Sci. Fenn. Math. 42 (2017).963-977.

16. T. Umezawa, Analytic functions convex in one direction, J. Math. Soc. Japan 4 (1952), 194-202.

17. H. S. Wall, Analytic Theory of Continued Fractions, D. Van Nostrand Co. Inc., New York, 1948.

School of Statistics, University of International Business and Economics, No. 10, Huixin Donguie, Chaoyang District, Beijing 100029, China

E-mail address: wangmabe1@163.com 